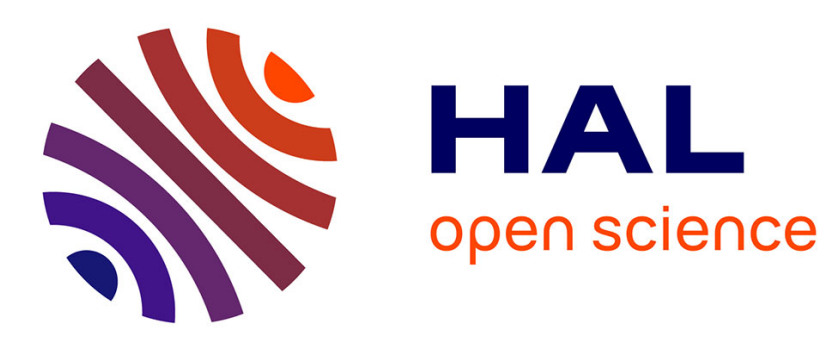

\title{
High-Precision Gluing Tasks Based on Thick Films of Glue and a Microrobotics Approach
}

Bhawnath Tiwari, Cédric Clevy, Philippe Lutz

\section{To cite this version:}

Bhawnath Tiwari, Cédric Clevy, Philippe Lutz. High-Precision Gluing Tasks Based on Thick Films of Glue and a Microrobotics Approach. IEEE Robotics and Automation Letters, 2019, 4 (4), pp.4370 - 4377. 10.1109/lra.2019.2932881 . hal-03335191

\section{HAL Id: hal-03335191 \\ https://hal.science/hal-03335191}

Submitted on 7 Feb 2022

HAL is a multi-disciplinary open access archive for the deposit and dissemination of scientific research documents, whether they are published or not. The documents may come from teaching and research institutions in France or abroad, or from public or private research centers.
L'archive ouverte pluridisciplinaire HAL, est destinée au dépôt et à la diffusion de documents scientifiques de niveau recherche, publiés ou non, émanant des établissements d'enseignement et de recherche français ou étrangers, des laboratoires publics ou privés. 


\title{
High-Precision Gluing Tasks Based on Thick Films of Glue and a Microrobotics Approach
}

\author{
Bhawnath Tiwari ${ }^{\circledR}$, Cédric Clévy ${ }^{\circledR}$, and Philippe Lutz
}

\begin{abstract}
Micro-assembly using glue is an industrial need, but at the microscale, the gluing process appears highly challenging. Especially when the thickness of the glue film is large relative to the size of components to be assembled. Moreover, the down-scaling of passive techniques used at the macroscale (such as using reference surfaces) are widespread, but require highly complex and dedicated procedures to reach the required precision (typically $1 \mu \mathrm{m}$ or below). To address this, we propose an original approach for micro-assembly that consists of using a thick film of glue (glue thickness typically $>\mathbf{5 0} \boldsymbol{\mu \mathrm { m }}$ ) with an active microrobotic control. This approach enables us to have high precision positioning of the two components during the gluing task. The proposed approach appears highly versatile and flexible because it is independent of many parameters that usually influence gluing tasks. Several experiments without the use of the proposed approach show that the typical order of positional drift from curing could be of 5 to $15 \mu \mathrm{m}$. Finally, experimental results using the proposed approach demonstrate that a positioning accuracy of $200 \mathrm{~nm}$ can typically be obtained, which validates the approach and suggests its potential interest to industry.
\end{abstract}

Index Terms-Automation at micro-nano scales, assembly, micro/nano robots.

\section{INTRODUCTION}

W ITH the growing requirements at the microscale, the demand for a precise microassembly has much grown. There were several approaches used and proposed for microassembly, depending upon the modes of operation required. In [1], a rapid aqueous process based self-assembly of nanoparticles has been presented, whereas the assembly of modular and polymer-based microfluidic devices has also been discussed [2]. Microassembly with very high accuracy has been demonstrated in several works, such as for optical components positioning [3] and assembly of flexible structures [4], [5], but gluing tasks for a micro-assembly have been little investigated, despite being very widespread [6].

Manuscript received February 24, 2019; accepted July 15, 2019. Date of publication August 2, 2019; date of current version August 15, 2019. This letter was recommended for publication by Associate Editor X. Liu and Editor D. Popa upon evaluation of the reviewers' comments. This work was supported in part by COLAMIR project (Contract "ANR-16-CE10-0009"), in part by the FrancheComté region, in part by the EIPHI Graduate School (contract "ANR-17-EURE0002"), and in part by the Robotex platform (Contract "ANR-10-EQPX-44-01"). (Corresponding author: Bhawnath Tiwari.)

The authors are with the Department of Automatic Control and MicroMechatronic Systems, FEMTO-ST Institute, University of Bourgogne FrancheComté, CNRS, F-25000 Besancon, France (e-mail: bhawnath.tiwari@femto-st. fr; cedric.clevy@femto-st.fr; philippe.lutz@femto-st.fr).

Digital Object Identifier 10.1109/LRA.2019.2932881
Gluing appears to be a key task of a microassembly process because it enables to definitely hold the relative position between two components. For most applications, the quality/positioning accuracy of the assembly directly impact the performance of the final product. Most of the existing gluing tasks require very dedicated approaches, especially to use thin films using surface referencing and conditioning [7], [8]. For gluing two objects, even using a conditioned thin glue layer of $\sim 20 \mu \mathrm{m}$ can introduce significant error towards desired assembly (the typical need is to reach a positioning accuracy below $1 \mu \mathrm{m}$ ). Depending on the requirements, the thick glue layer can provide a wide range of desired distance between the two surfaces. In [9], $30 \mu \mathrm{m}$ thick conductive glue was used to connect a MEMS structure directly to a printed circuit board. Different applications of packaging, such as fiber fixation with a chip, might be made using a thick glue instead of using fusion splicing based dedicated approach, as used in [10], but positioning accuracy then needs to be insured by some other flexible means.

Surfaces in contact with adhesive make a huge contribution to the behavior during curing, especially their influence towards spreading coefficient and rate of spreading for the equilibrium stabilization [11]. Moreover, the choice and quantity of glue are also an important concern [12], [13]. Many other parameters, such as type of glue, glue quantity, surface roughness, surface shape, working temperature, and method of hardening the glue might influence the final positioning accuracy. The procedures needed reach high positioning accuracy must account for these parameters and their potential interactions. Therefore, there is a strong requirement of a flexible and versatile approach that could solve the complexity with sub-micrometer accuracy. To address this, we propose to use a thick film of glue (rather than the conventional thin film) combined with an active robot control strategy (rather than conventional passive approaches). Indeed, this approach appears particularly generic, versatile, and flexible because it intends to adapt to all of the mentioned-above parameter changes. In addition, using a thick film of glue might introduce significant positional drifts, primarily from capillary effects from surface-liquid glue interaction, followed by positional drift because of stress development during glue curing. To study this behavior, we first propose a generic system and associated control scheme to experimentally study the assembly strategy proposed (Section II). To demonstrate the potential of the approach, Section III will compare the typical positioning errors that might be induced by gluing processes with/without adopting the proposed strategy. Finally, conclusions and prospects are discussed in Section IV. 


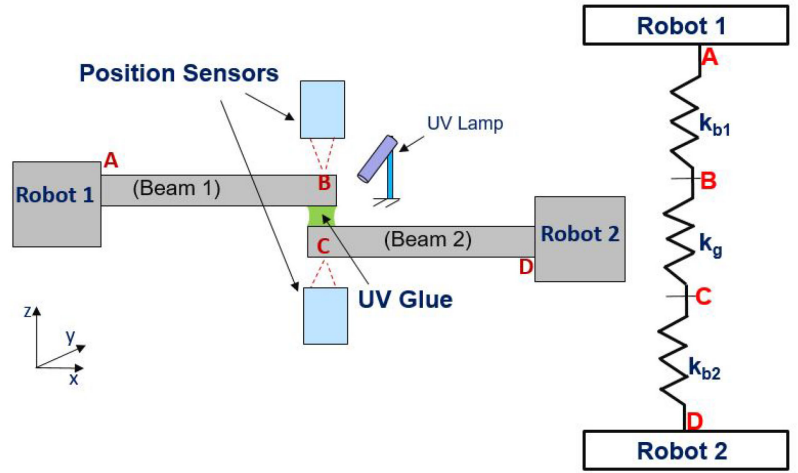

Fig. 1. Proposed Beam based system for analysis.

\section{EXPERIMENTAL SYSTEM PRINCIPLE AND CONTROLler DESIGN}

This section aims to introduce the experimental set-up and its associated control scheme, which will be used to investigate the original approach for gluing tasks at the microscale. This principle aims to enable the quantification of the positioning accuracy evolution during the gluing process, but also to investigate the improvement with the proposed approach, and to demonstrate its robustness.

\section{A. Principle of the Experimental System}

Glue curing introduces stress on the different surfaces to be glued. This stress development can introduce significant positional drift to the surfaces to be glued, which is a serious problem for the precise positioning of the surfaces. This work intends to address the problem of positioning accuracy resulting from glue curing and to introduce a versatile solution to meet positioning requirements. In the present work, gluing about the Z-axis (Fig. 1) is addressed using the presented beam-based system.

There are many parameters that can influence the assembly behavior, including glue quantity, relative contact area, and beam stiffness. The influence of the positional drift from glue curing about other planar ( $\mathrm{x}$ and $\mathrm{y}$ ) axes can be considered negligible relative to the z-axis, which was the case in [14]. This can be seen in context of tethered plane which is xy plane (contact with glue) in the presented configuration, so the orthogonal $\mathrm{z}$ axis would include the dominant shrinkage tensor, therefore the possibility of sliding in xy plane is negligible. The other problem is from a very intense UV exposure that might introduce small voids around the neutral axis of the beams [15]. This is mainly possible when using very fast curing of glue, so that neither the glue nor the beam surfaces can equilibrate rapid enough to restrict the stress development.

In the literature, there are several works reporting the use of "depth curing" in place of "surface curing" using photoinitiators [16]. With the suitable concentration and choice of photoinitiator, it could be possible to avoid the creation of void by facilitating the stress release. In the current work, the UV exposure intensity was minimized by placing the source suitably far (based on experimental observation) from the glue, thereby resulting in a slow curing rate, and the possibility of void creation could be minimized because of sufficient time for stress release. The other justification for negligible void creation can be explained by the length to width ratio of the employed beams, which is more than 20 times for all chosen beams. So, overall, the major effect is considered to be along the longitudinal z-axis. The idea is to demonstrate the validity of the proposed approach about the chosen axis, which can then be extended to another axis (with the inclusion of an additional sensing mechanism).

The top beam can be, for instance, a microgripper holding a micro-object (part in contact with the glue) and the bottom beam as a substrate. Basically, the presented configuration and the opted approach was chosen in the context of a tethered configuration for microassembly. The objective will be to control the relative distance [i.e., the distance between the two beams tips (denoted as points B and C of Fig. 1)].

$$
\begin{aligned}
& F_{A}=k_{b 1} \times\left(\delta_{A}-\delta_{B}\right) \\
& F_{B}=k_{g} \times\left(\delta_{B}-\delta_{C}\right) \\
& F_{D}=k_{b 2} \times\left(\delta_{C}-\delta_{D}\right)
\end{aligned}
$$

In the presented model of Fig. 1, the displacement of points A, $\mathrm{B}, \mathrm{C}$, and D (along the z-axis) are $\delta_{A}, \delta_{B}, \delta_{C}$ and $\delta_{D}$ respectively. Beam 1 stiffness is termed as $k_{b 1}$ and Beam 2 stiffness as $k_{b 2}$, whereas the glue stiffness is termed as $k_{g} . F_{A}, F_{B}, F_{C}$ and $F_{D}$, which are the forces acting at point A (eqn. (1)), B (eqn. (2)), C, and D (eqn. (3)) respectively. $F_{B}$ and $F_{C}$ are expected to be numerically equal and also under stabilized equilibrium; all forces should be equal because of the chain configuration.

\section{B. Controller Design}

As glue curing process induces mechanical stress and so a drift, there is a requirement of an adequate action that can restrain this additional drift. There could be several approaches to solve this mismatch. In [17], shrinkage compensation from UV-curing was addressed using identifying critical parameters of curing. This identification was made by numerical simulation with knowledge of the sensitivity of the isolated degree of freedom, and accordingly, bonding direction was used. But this approach has its limitations: primarily from the choice of glue, and secondly from its convenient adaptability towards the microassembly process. Another possible approach could be to manage the viscosity variation to restrain the possible reshaping of glue arising from the glue curing. In [18], one apparatus is proposed with which viscosity adjustment of UV adhesive could be made. But mapping up the viscosity adjustment with the microassembly might further limit the workspace and the strategy towards assembly, as there might be other factors (such as choice of glue and cured strength) that complicate the chosen approach. With the knowledge of physical happening and its use in the approach it could be possible to further improve the flexibility of the approach (towards force/position-based control).

There are multiple ways to address this problem; one would be to go with the approach of force/position control. To solve 


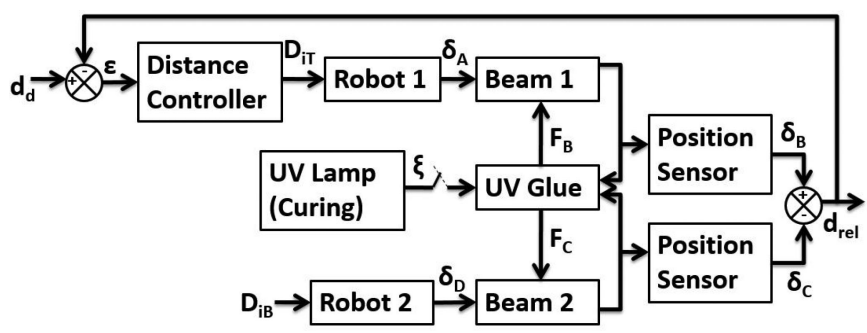

Fig. 2. Proposed scheme for distance control between the Beams.

the problem of desired relative distance resulting from positional drift, one approach could be to track force/position, and accordingly generate sufficient force against the glue curing, thereby avoiding this drift. This case is quite similar to that of grippers end-effector against the dynamic environment. An impedance control-based approach to estimate the unknown environments parameter using force tracking is presented in [19]; the approach was tested with different compliant structures. In [20], the force sensor was used to detect the penetration force against a biological cell, and a strain-based sensor to measure the relative position of the micropipette. With knowledge of force/position, an incremental PID controller was proposed to meet the suitable injection force. Another similar situation could be the case where the teleoperation task needs to be done against the dynamic environment, resulting in a requirement of force/position knowledge to develop suitable behavior for the desired teleoperation. This case was addressed in [21], where a fuzzy-observer based hybrid force/position control method was used for a bimanual teleoperation system in the presence of dynamic uncertainties.

Combining these several studies, in the context of glue curing, an active control-based strategy can be used to control the distance between the beams, where Beam 1 would be allowed to find a suitable position to have sufficient force at the assembly end, through which the desired distance between the beams can be obtained.

The proposed active control strategy is shown in Fig. 2. There are two robots, Robot 1 and Robot 2, which are connected to Beam 1 and Beam 2, respectively, but only Robot 1 was used for the control of distance. Robot 2 has been placed at fixed position $D_{i B}$ with constant input to the Robot for entire experimentation, and is therefore not directly included in feedback control. The position of the beams is influenced by their respectively connected robots, and also by contact with the UV glue placed in between. The respective beam positions would also be influenced by the presence of UV light (turning on the UV lamp); this additional influence has been denoted as $\xi$. All of the measured positions shown in the control scheme (Fig. 2) are in common frame. The actual relative distance between the beams $\left(d_{r e l}\right)$ must be compared with the desired distance between the beams $\left(d_{d}\right)$ to generate the error signal $\varepsilon$, which is further processed by a proportional Distance Controller with gain $(\mathrm{K})$. The output $D_{i T}$ from the controller was input to Robot 1 , thereby achieving the necessary changes in its position. With the internal sensor of the robot, the current position and the desired change were compared to make the necessary updates.

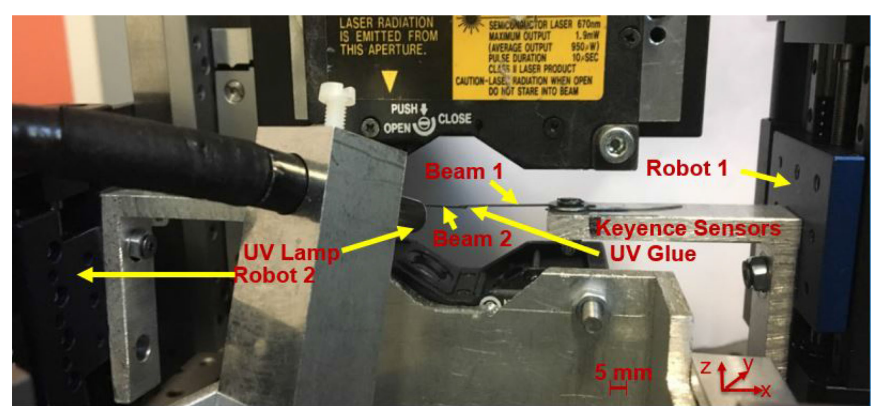

Fig. 3. Experimental Setup used for the analysis.

\section{EXPERIMENTAL WORK}

There are two main requirements from the experimental side. First, to be able to analyze the glue curing behavior, as discussed in Section II-A. Second, to be able to perform the defined active control action explained in Section II-B.

\section{A. Experimental Setup}

The experimental setup based on the proposed beam model (Fig. 1) is shown in Fig. 3. There are two Keyence (LC-2420) position sensors utilized, which could have sensing resolution up to $10 \mathrm{~nm}$ (under defined standard condition by Keyence), and are centered along the neutral axis of the respective beams at the assembly end. Keyence sensors work by a laser reflection principle, through which the respective beams bending or movement can be measured.

There are three kinds of beams utilized throughout the different experiments, all made of steel material with the stiffness of 10,20 , and $167.5 \mathrm{~N} / \mathrm{m}$ to investigate different potential behaviors that may happen during a gluing task. The targeted area (the shared area between beams) for gluing throughout the different experiments was $1 \times 1 \mathrm{~mm}$. The chosen UV glue for the experimentation was VITRALIT 6128, whereas the UV light source utilized for glue curing was DYMAX Blue Wave 50 with UV emission of $365 \mathrm{~nm}$ wavelength. As shown in the experimental setup, the two beams are attached to Robots, these are basically M-122-2DD motors from Physik Instrumente (PI), and the used controllers for these motors were C-863 from Mercury. Along with these key elements of the presented experimental setup, there were mechanical stages, which were used for the pre-positioning of different elements.

\section{B. Behavior in the Absence of the Proposed Control Scheme}

In this section, several experimental results are investigated to analyze the challenges in the absence of the proposed control scheme. Because of the use of thick film, capillary action should also have an influence on the positioning.

To monitor the behavior during glue contact, Beam 1 and Beam 2 of $167.5 \mathrm{~N} / \mathrm{m}$ was used. In Fig. 4, the initial glue droplet size is approximately $300 \mu \mathrm{m}$, and Beam 1 is targeted to be placed $100 \mu \mathrm{m}$ away from Beam 2. For approximately first $10 \mathrm{~s}$, Beam 1 was in free motion and then it came in contact with glue. With glue-beam contact, Beam 1 followed a constrained motion from time 10 to $16 \mathrm{~s}$. During the phase of constrained 


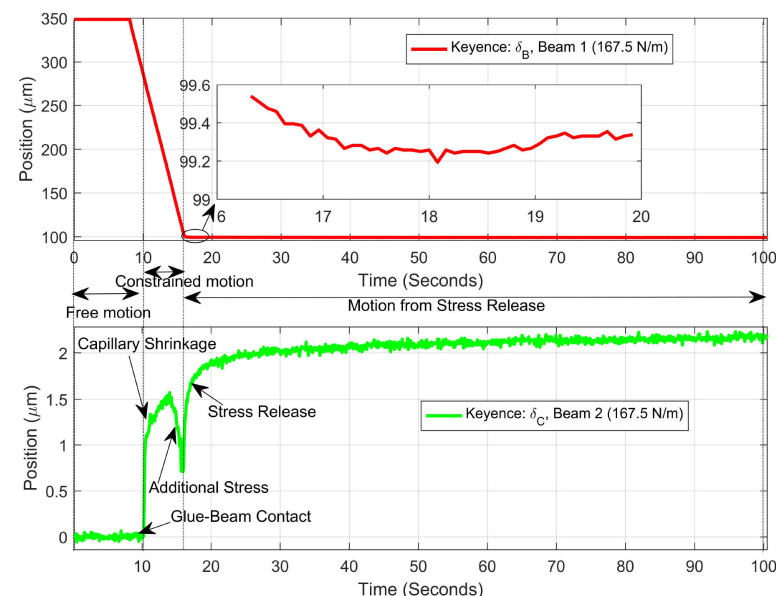

Fig. 4. Glue contact using $167.5 \mathrm{~N} / \mathrm{m}$ of Beam 1 and Beam 2.

motion, a capillary based shrinkage can be seen, which means the stress resulted from Beam 1 movement was not stronger than the capillary stress and so this resulted into Beam 2 bending towards Beam 1. But there is a time when the stress from Beam 1 pressing became significantly higher than the capillary stress (marked zone as "Additional Stress" in Fig. 4), this would result into Beam 2 motion along the same direction as that of Beam 1. The developed stress starts to release as soon the introduced pressing from Beam 1 is stopped. The quantity of glue and extent of stress developed would influence the final positioning of the end of the beam. Therefore, there is considerable drift possible from the capillary action during the beam placement against the glue. There are multiple factors working together at the liquid phase of the glue, adding up multiple possible uncertainties for final positioning of the beams. This positional drift may be minimized by suitable placement of the Beam 1 against the glue, but the major influence is from the positional drift induced during glue curing.

To start with glue curing, a wait of a certain duration was made to allow the stabilization of both beams against the liquid glue. This stabilization was considered when the drift minimized to the order of sensing resolution. In Fig. 5, Beam 1 (of stiffness $167.5 \mathrm{~N} / \mathrm{m}$ ) is used against Beam 2 (of $167.5 \mathrm{~N} / \mathrm{m}$ stiffness). Beam positions are at a constant level before the start of UV light, with an initial distance of $101.6 \mu \mathrm{m}$. As a result of turning on the UV light, the two beams deform towards each other, with drifts of $4.1 \mu \mathrm{m}$ and $11 \mu \mathrm{m}$ from Beam 1 and Beam 2, respectively. A slow drift after the first quick drift (just after UV $\mathrm{ON}$ ) is mainly because of the stress release derived from initial press/bending of the beams.

As the primary interest is to maintain the desired distance between the beams, the drift induced by glue curing is addressed through shrinkage of the distance (between the beams). There were several experiments performed (listed in Table I) to examine the shrinkage order between the beams. The distances between the beams before the start of glue curing are listed as " $G_{i}$ " in Table I, whereas the distance as a result of glue curing is listed as " $G_{f}$ ". Therefore, the shown Distance Drift (Difference of $G_{i}$ and $G_{f}$ ) in the table is mainly from the glue

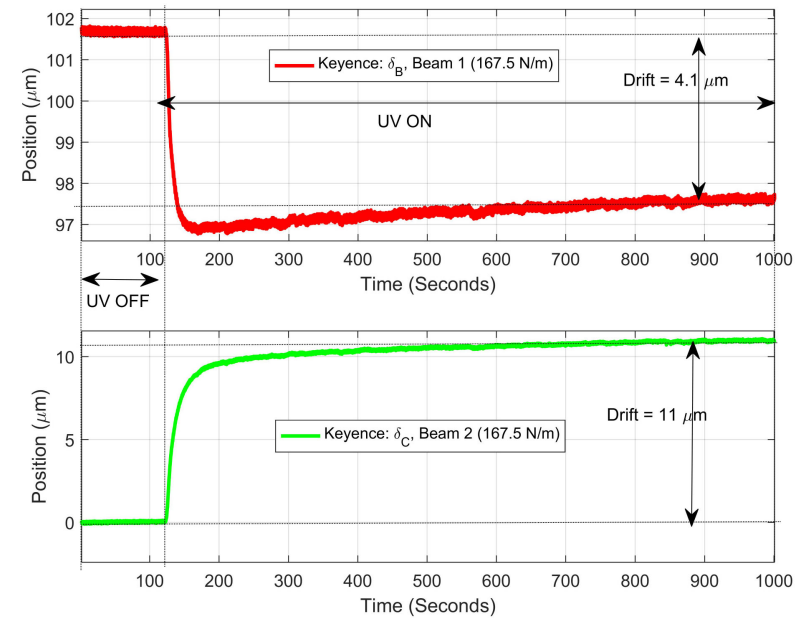

Fig. 5. Glue curing using $167.5 \mathrm{~N} / \mathrm{m}$ of Beam 1 and Beam 2 with initial gap of $101.6 \mu \mathrm{m}$

TABLE I

OBTAINED SHRINKAGE FROM DIFFERENT EXPERIMENTS

\begin{tabular}{ccccc}
\hline \hline$k_{b 1}(\mathrm{~N} / \mathrm{m})$ & $k_{b 2}(\mathrm{~N} / \mathrm{m})$ & $G_{i}(\mu \mathrm{m})$ & $G_{f}(\mu \mathrm{m})$ & Distance Drift $(\mu \mathrm{m})$ \\
\hline 167.5 & 10 & 99.2 & 88.9 & 10.3 \\
167.5 & 10 & 93.25 & 78.9 & 14.35 \\
167.5 & 20 & 88 & 76.6 & 11.4 \\
167.5 & 20 & 68.8 & 63.1 & 5.7 \\
167.5 & 167.5 & 101.6 & 86.5 & 15.1 \\
167.5 & 167.5 & 98.5 & 86.5 & 12 \\
\hline
\end{tabular}

curing, whereas we do have the shrinkage from the capillary action dependent on introduced stress and glue quantity (as seen in Fig. 4). The big difference in distance drifts from 11.4 to $5.7 \mathrm{~m}$ in two runs with $20 \mathrm{~N} / \mathrm{m}$ for Beam 2 is mainly because of the different glue quantities between the beams. The amount of drift is directly dependent on the quantity of glue. The experimental results are shown in Table I, which differ mainly because glue quantity was not controlled in different parallel runs. This also demonstrates the behavior sensitivity over different parameters. The shrinkage from capillary action (before the start of curing) ranged between 2 and $17 \mu \mathrm{m}$, throughout the different experiments listed in the Table I. Therefore, the overall shrinkage from the gluing process could reach up to $30 \mu \mathrm{m}$, which is a serious concerned from an assembly accuracy point of view. Therefore, the key objective of this work is to have a versatile approach for microassembly using UV glue, which could result in a repeatable sub-micrometer accuracy positioning.

\section{Behavior With Proposed Control Strategy}

The chosen control scheme (Fig. 2) with a single robot allows the flexibility for microassmebly operation, especially where the substrate is not robotized. The intention in the proposed work is to adopt a versatile approach that can also work for a thick film (a worse case than with thin films), which is targeted to avoid the special dedicated treatment for gluing. Glue thickness $>50 \mu \mathrm{m}$ is targeted to demonstrate the feasibility of the approach, but the approach aims at being independent of the choice 


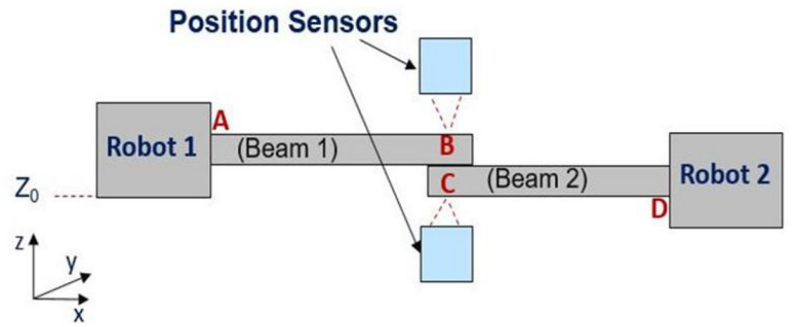

Fig. 6. Step 1, Used for $Z_{0}$ identification.

of glue thickness. Obviously, a thick glue would require higher drag force (than thin film) dependent on glue quantity and the shared area between the glue and contact surfaces. The limits in terms of glue quantity and the area that can be glued can be defined from sensing and opted systems limitation. For example, in the presented case, Keyence position sensors were used, to be in the sensing range a relatively high stiffness beam (chosen $167.5 \mathrm{~N} / \mathrm{m}$ ) as compared to 10 or $20 \mathrm{~N} / \mathrm{m}$ was used. As with stiffer beam, a higher force can be generated at the assembly end with small movement of the beam whereas in case of 10 or $20 \mathrm{~N} / \mathrm{m}$ beam, beams need to move out of the Keyence sensing range dependent on chosen glue size and area to be glued. Moreover, the chosen stiffness is closed to the stiffness of instrumented gripper $(130 \mathrm{~N} / \mathrm{m})$ used in [22], therefore a comparative analysis in terms of limitation to tasks handling could also be done.

The main principle of the control strategy employed is to move Robot 1 with sufficient force at the assembly end (beams end) so that the desired distance between the beams can be obtained. The passive beams position is important information which is required for processing of the control action. This position would be influenced depending on the beams interaction with glue and the movement from Robot 1 . Therefore, the entire experiment has been divided into five steps. Step 5 includes the proposed active control strategy, whereas the other steps are used to track the positional changes during the different phases of the gluing process.

1) Calculation of the Bottom Beams Relative Position: The chosen Keyence sensor for position sensing has a typical sensing range of $400 \mu \mathrm{m}$, and therefore the targeted starting distance was chosen to be in this range. Also, based on the direction of movement of beams, the respective Keyence sensors were positioned close to the extreme sensing value. For an efficient implementation of control strategy, there is a requirement for the calculation of all positions in a common frame. Therefore, the Beam 2 position (point $\mathrm{C}$ ) was chosen as the world frame for all experimentation. The Beam 2 position, taken as the origin, is symbolically termed as $Z_{0}$. An experimental model to show the interactions between the beams is shown in Fig. 6.

A trapezoidal signal (amplitude, $300 \mu \mathrm{m}$ ) was given to the Robot 1 (Fig. 7) to allow Beam 1 movement towards Beam 2. And based on the movement of Beam 2 under the interaction with Beam 1, the Beam 2 position can be calculated. This leads to the identification of position $Z_{0}$ relative to Robot 1 movement.
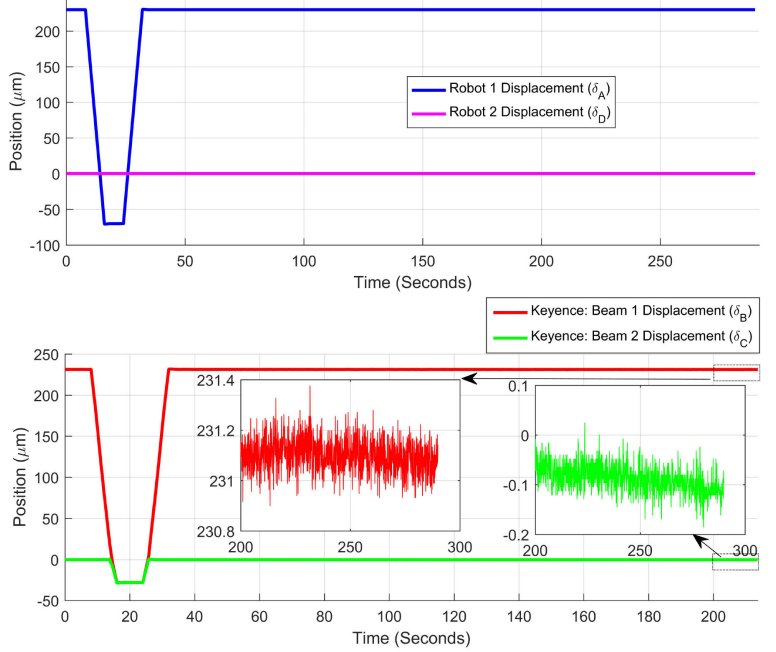

Fig. 7. The relative position of Beams identification using trapezoidal input to Robot 1 (Step 1).

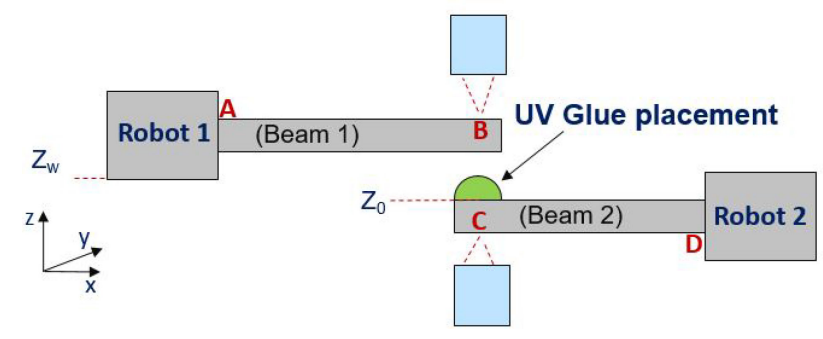

Fig. 8. Step 2, Glue Placement at Beam 2.

2) Glue Deposition: Because of the possible lack of elastic behavior, it is possible that, because of gluing or placement tool interaction, the substrate (Beam 2) moved to another stable position. Therefore, to be sure about the happenings throughout the phase of the glue placement, the position of Beam 2 was monitored. To have sufficient space for glue placement at Beam 2 , Robot 1 was moved to a waiting position $Z_{w}$, and the Beam 2 position was accordingly monitored. In Fig. $9, Z_{w}$ was $4 \mathrm{~mm}$, and an impulsive displacement can be seen as a result of interaction between the Beam 2 and gluing tool.

3) Contact of Beam 1 With Glue and Stabilization: Now there is a requirement of placing the Beam 1 in contact with glue (Step 3), but at the same time, there could be a considerable spreading of glue (Fig. 10). Based on the identified $Z_{0}$ from Step 1 , and the targeted distance between the beams $\left(d_{d}\right)$, which is $50 \mu \mathrm{m}$, Robot 1 was sent to a position $Z_{0}+50 \mu \mathrm{m}$. Dependent on the viscosity, quantity of glue, stiffness of the beams, and applied pressure around the beams, the actual distance between the beams might be different than the targeted $50 \mu \mathrm{m}$. In Fig. 11, the beginning of Step 3 is at a constant level in the absence of glue contact with Beam 1; then, as a result of its contact and further movement with glue, Beam 2 is compressed by $4.5 \mu \mathrm{m}$. Once Beam 1 is allowed to stay at the same level after completing the initial movement (Step 3), Beam 2 moved towards Beam 1, which was a result of capillary action together with the release 

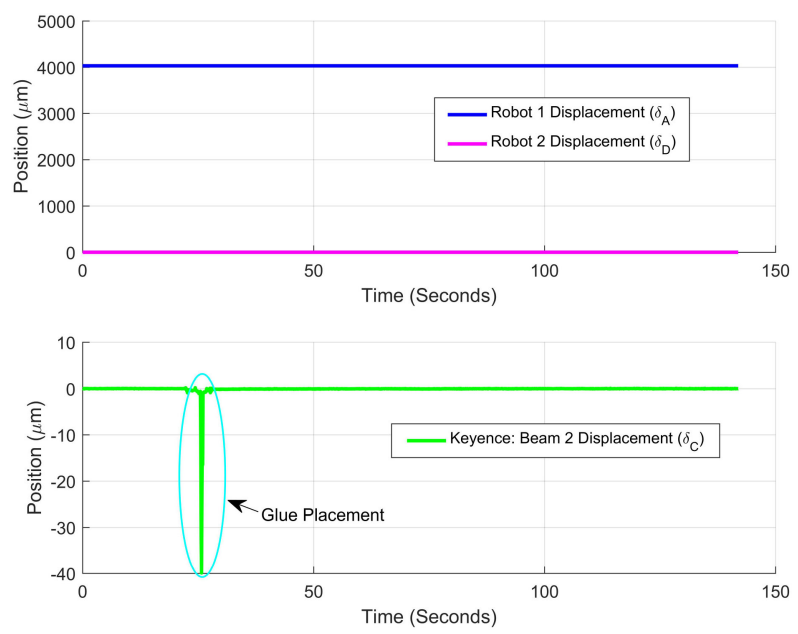

Fig. 9. Behavior through glue placement phase at Beam 2 (Step 2).

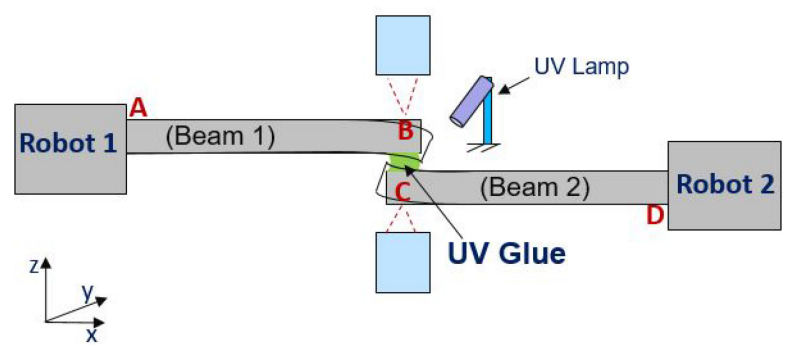

Fig. 10. Steps 3 and 4, Beam 1 contact with the Glue and Glue stabilization.

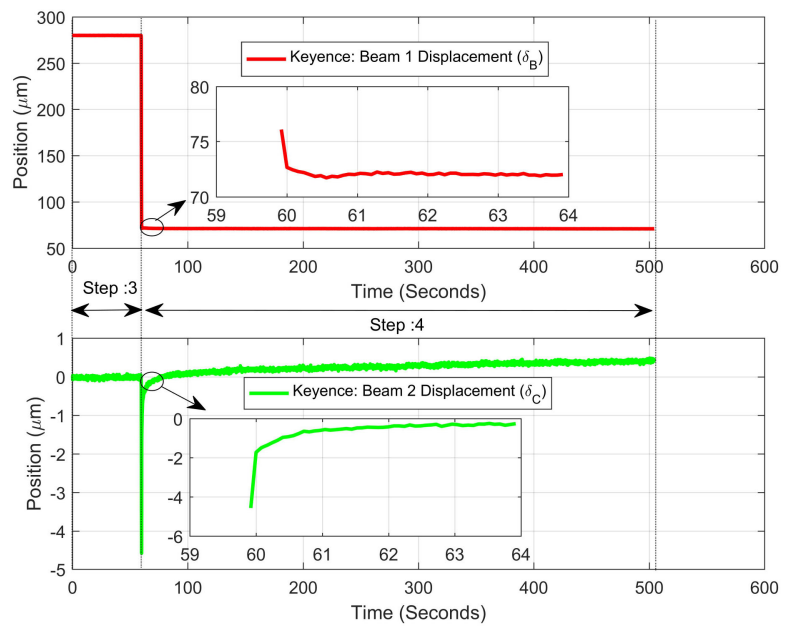

Fig. 11. Obtained behavior through Beam 1 contact with Glue and Glue stabilization (Steps 3 and 4).

of the introduced stress. The respective beams movements were monitored until acceptable stability (of the order of sensing resolution) was obtained (Step 4).

4) Glue Curing and Distance Control: From Fig. 7, it can be seen in the zoomed-in part that even in the absence of any movement from the robot, and glue/beam interaction, there is the presence of oscillation which is, in fact, the noise from

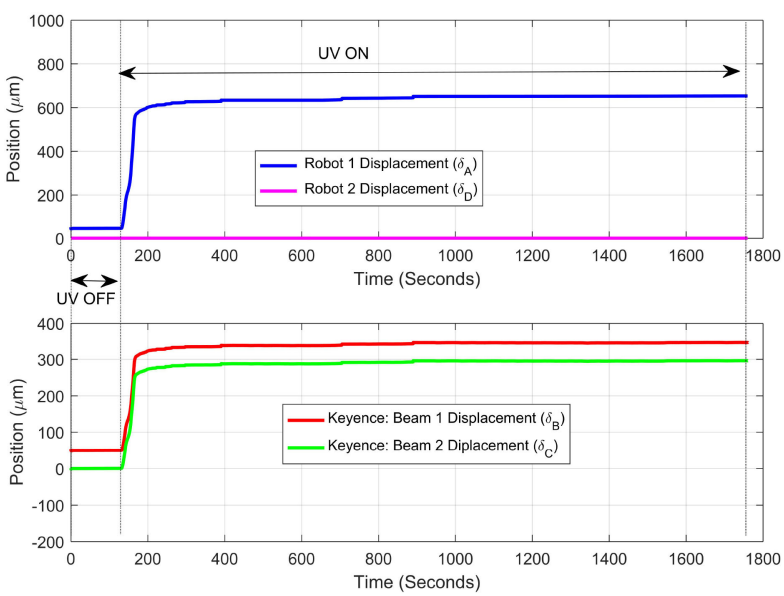

Fig. 12. Step 5, Control action with Glue Curing.

different sources of mechanical vibrations around. This signifies the limitation of the system in terms of noise influence, so with the use of distance controller, the error signal $(\varepsilon)$ falling in the range of noise amplitude may not be trusted. Therefore, the correction against the order of noise can be ignored. This could provide two main benefits. First, the controller would be stabilized when there is no need for correction. Second, because of the controller stabilization, the cured link between the two beams will achieve relatively good strength compared to that against continuous correction from the controller. Also, Robot 1 and Robot 2 have the minimum step movements of $0.2 \mu \mathrm{m}$. Therefore, taking into consideration the system limitations and noise influence, a dead zone of $\pm 0.15 \mu \mathrm{m}$ is included with the distance controller. So, if the error signal $(\varepsilon)$, as shown in Fig. 2, is inside the defined dead zone range, then there will be no correction in position $D_{i T}$ of the Robot 1 .

Before glue curing starts, it is important to ensure an initial condition, such as the stable positioning of the beams in the absence of UV, and then UV light can be turned ON to start the gluing task (Step 5). For the processing of the experiment, the Baud rate of 38400 was used for communication to Robots using mercury C-863 controller (based on PID). To achieve suitable speed and avoid overshoots (sensing range limitation), the Distance Controller (Fig. 2) chosen was a proportional controller with unity gain. For the exchange of signals, a NI USB-6353 card was used. The obtained sampling frequency for different processed signals was $12.5 \mathrm{~Hz}$. As the included Keyence sensors are external to the glue-beam system and, therefore, the position of the sensor was positioned well to be within convenient sensing range for the entire assembly operation.

The multiple sources of uncertainty seen in Section III-B coming from capillary effect (liquid glue) and glue curing are combinedly seen as a single perturbation to the system. The proposed control scheme has to reject this perturbation for the desired behavior. In Fig. 12, Beam 1 moved by $300 \mu \mathrm{m}$ to generate sufficient force between the beams to have the desired distance $\left(d_{d}\right)$ of $50 \mu \mathrm{m}$. From eqn. 1, with the beam stiffness of $167.5 \mathrm{~N} / \mathrm{m}$, the order of force required to achieve the desired distance is $50 \mathrm{mN}$. The key requirement is the sufficient force 


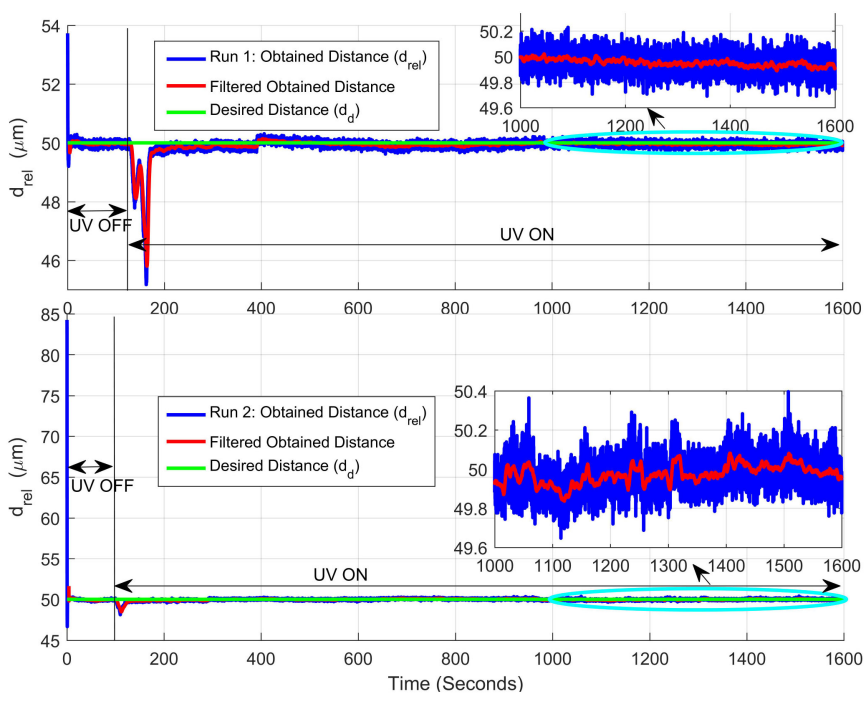

Fig. 13. Step 5, Obtained behavior $\left(d_{r e l}=\delta_{B}-\delta_{C}\right)$ for $d_{d}=50 \mu \mathrm{m}$.

at the assembly end, and this requirement was fulfilled by the automated movement of Beam 1 as a result of the proposed control scheme. From Fig. 12, it can also be seen that the major distance correction against the curing was made in the first $60 \mathrm{~s}$ after turning on the UV light. Therefore, the proposed control action is fast enough to adapt the requirements against the curing.

The obtained distance between the beams oscillates slightly near this defined dead zone value. Fig. 13 highlights the distance evolution over time during this gluing process. There were several experiments done to test the repeatability of the approach, and the experimental result from the two runs (Run 1 and Run 2) is shown in Fig. 13. Run 1 corresponds to Fig. 12, whereas Run 2 corresponds to another experimental result. Before switching on the UV light (in Run 1 of Fig. 13), the initial distance between the beams was controlled from 53.9 to $50 \mu \mathrm{m}$. The proposed control action can maintain the desired distance with an error in the order of $200 \mathrm{~nm}$. By saying order $200 \mathrm{~nm}$ means, the obtained distance signal $\left(d_{r e l}\right)$ oscillates (with the exclusion of spikes) by $200 \mathrm{~nm}$ around the targeted distance. But it can be seen that with the use of a moving average filter, the filtered signal is very close to the desired $50 \mu \mathrm{m}$ (typically less than $20 \mathrm{~nm}$ ). There are likely multiple sources of this noise; the main sources could be the mechanical vibration resulting from the electrical devices around, the different vibrations from the movement around, and also the optical presence might have a contribution to the obtained noise. In Run 2, the initial distance between the beams was $84.6 \mu \mathrm{m}$, but with the proposed approach the final distance between the beams is of the same order as that of Run 1, indicating the repeatability of the behavior. From Fig. 12, there is no movement of Robot 1 after $\sim 900 \mathrm{~s}$, which means the error in desired and the actual distance between the beams is well inside the defined dead zone. Therefore, the noted dynamics after stopping the movement of Robot 1, as shown in the zoomed-in version of Fig. 13, mainly comes from the noise. Achieving accuracy around the opted dead zone and the resolution of the sensor also indicates that, if the chosen systems limitation can be removed or minimized, then it could be possible to further improve the accuracy with the same approach for the distance control.

\section{CONCLUSIONS}

The proposed study addresses the challenges of microassembly with glue, especially the drift from glue curing. There was no special surface treatment used for the assembly. Moreover, the approach opted was independent of the choice of glue, which makes this approach flexible and versatile. The chosen approach combining thick film of glue with an active robot control strategy has demonstrated sub-micrometer high positioning accuracy in the order of $200 \mathrm{~nm}$ (filtered $20 \mathrm{~nm}$ ) between the two components to be glued. This drift could typically reach $15 \mu \mathrm{m}$ without the use of the proposed control approach. With the applied control actions proposed, the typical order of force required to have the desired behavior was $50 \mathrm{mN}$. With knowledge of the order of positional drifts and required force, appropriate sensing and gripping tool choices can also be made for microassembly using glue.

The presented work also appears helpful towards microassembly system design for gluing tasks. To add further flexibility to the opted approach, it becomes important to address the order of misalignment because of the void created during fast UV curing. This approach should not be further dependent on the choice of stiffness around (length to width ratio). To meet these requirements, as future work, we would like to apply the approach for a further precise assembly of micro-object using an instrumented gripping tool capable of multi-degrees of freedom, sensing, and handling.

\section{REFERENCES}

[1] S. Cui et al., "Aqueous assembly of oxide and fluoride nanoparticles into 3D microassemblies," Langmuir, vol. 34, no. 27, pp. 8075-8080, 2018.

[2] T. Y. Lee, K. Han, D. O. Barrett, S. Park, S. A. Soper, and M. C. Murphy, "Accurate, predictable, repeatable micro-assembly technology for polymer, microfluidic modules," Sensors Actuators, B Chem., vol. 254, pp. 1249-1258, 2018.

[3] H. Bettahar, A. Caspar, C. Clévy, N. Courjal, and P. Lutz, "Photo-robotic positioning for integrated optics," IEEE Robot. Autom. Lett., vol. 2, no. 1, pp. 217-222, Jan. 2017

[4] B. Komati et al., "Automated robotic microassembly of flexible optical components," in Proc. IEEE Int. Symp. Assem. Manuf., 2016, pp. 93-98.

[5] D. Xing, F. Liu, S. Liu, and D. Xu, "Efficient insertion of partially flexible objects in precision assembly," IEEE Trans. Autom. Sci. Eng., vol. 16, no. 2, pp. 706-715, Apr. 2019.

[6] R. J. Chang and C. C. Chen, "Using microgripper in development of automatic adhesive glue transferring and binding microassembly system," Engineering, vol. 2, no. 1, pp. 1-11, 2010.

[7] Y. H. Tennico, M. T. Kosedjojo, S. Kondo, D. T. Mandrell, and V. T. Remcho, "Surface modification-assisted bonding of polymer-based microfluidic devices," Sensors Actuators B, Chem., vol. 143, no. 2, pp. 799-804, 2010.

[8] L. Xiong, P. Chen, and Q. Zhou, "Adhesion promotion between PDMS and glass by oxygen plasma pre-treatment," J. Adhesion Sci. Technol., vol. 28, no. 11, pp. 1046-1054, 2014.

[9] J. Gakkestad, P. Dalsjo, H. Kristiansen, R. Johannessen, and M. M. V. Taklo, "Use of conductive adhesive for MEMS interconnection in ammunition fuze applications," J. Micro/Nanolithography, MEMS, MOEMS, vol. 9, no. 4, 2010, Art. no. 041108.

[10] J. Nauriyal, M. Song, R. Yu, and J. Cardenas, "Fiber to chip fusion splicing for robust, low loss photonic packaging," Proc. Conf. Lasers Electro-Opt, p. JW2A.72, 2018. 
[11] L. F. M. da Silva, A. Ochsner, and R. D. Adams, Handbook of Adhesion Technology. Berlin, Germany: Springer-Verlag, 2011.

[12] R. D. Adams and W. C. Wake, "Factors influencing the choice of adhesive," in Structural Adhesive Joints in Engineering. New York, NY, USA: Springer, 1984.

[13] J. Y. Cognard, R. Creachcadec, L. Sohier, and D. Leguillon, "Influence of adhesive thickness on the behaviour of bonded assemblies under shear loadings using a modified TAST fixture," Int. J. Adhesion Adhesives, vol. 30, pp. 257-266, 2010.

[14] J. Dai, S. Xi, and D. Li, "Numerical analysis of curing residual stress and deformation in thermosetting composite laminates with comparison between different constitutive models," Materials, vol. 12, no. 4, 2019, Art. no. E572.

[15] E. A. Norland and F. S. Martin, "Mechanisms relating to reducing stress in curing thick sections of UV adhesives," Norland Products Inc., North Brunswick, NJ, USA, 2000.

[16] H. Okamura, S. Niizeki, T. Ochi, and A. Matsumoto, "UV curable formulations for UV-C LEDs," J. Photopolym. Sci. Technol., vol. 29, pp. 99-104, 2016.
[17] T. Müller et al., "Strategies for precision adhesive bonding of micro-optical systems," Proc. SPIE, vol. 9346, 2015, Art. no. 93460E.

[18] R. Roajanasiri and B. Buasak, "UV adhesive viscocity adjustment apparatus and method," United States Patent US825 627 2B1, 2012.

[19] B. Komati, C. Clévy, and P. Lutz, "Force tracking impedance control with unknown environment at the microscale," in Proc. IEEE Int. Conf. Robot. Autom., 2014, pp. 5203-5208.

[20] G. Wang and Q. Xu, "Design and precision position/force control of a piezo-driven microinjection system," IEEE/ASME Trans. Mechatronics, vol. 22, no. 4, pp. 1744-1754, Aug. 2017.

[21] Z. Lu, P. Huang, Z. Liu, and H. Chen, "Fuzzy-observer-based hybrid force/position control design for a multiple-sampling-rate bimanual teleoperation system," IEEE Trans. Fuzzy Syst., vol. 27, no. 7, pp. 1383-1396, Jul. 2019.

[22] B. Komati, J. Agnus, C. Clévy, and P. Lutz, "Prototyping of a highly performant and integrated piezoresistive force sensor for microscale application," J. Micromech. Microeng., vol. 24, 2014, Art. no. 035018 . 